\title{
Pancreas and kidney transplantation: the San Raffaele Hospital (Milan, Italy) experience
}

\author{
V. Di Carlo ${ }^{1}$, C. Staudacher ${ }^{1}$, M. Cristallo ${ }^{1}$, G. Ferrari ${ }^{1}$, M. Carlucci ${ }^{1}$, R. Castoldi $^{1}$, A. Secchi ${ }^{2}$, E. La Rocca ${ }^{2}$, \\ S. Martinenghi ${ }^{2}$, R. Caldara ${ }^{2}$, G. Gallioli ${ }^{3}$, C. Martani ${ }^{3}$, G. Torri ${ }^{3}$ and G. Pozza ${ }^{2}$ \\ ${ }^{1}$ Cattedra di Patologia Chirurgica, ${ }^{2}$ Department of Medicine, ${ }^{3}$ Department of Anaesthesiology and Intensive Care Unit, University of \\ Milan, San Raffaele Hospital, Milan, Italy
}

Summary.Results of 33 simultaneous pancreas and kidney transplantations performed at the San Raffaele Hospital, Milan, Italy are presented. In 26 cases segmental neoprene duct-injected grafts were transplanted and in seven cases, duodenopancreatic bladder-drained grafts. Five-year patient, kidney and pancreas survival were respectively, $89 \%, 72 \%$ and $58 \%$. Five-year survival in patients with technically successful pancreas transplants was $73 \%$. Thrombosis occured in $20 \%$ of cases. Mortality was $6 \%$ and overall morbidity $76 \%$. Surgical complications were present in $51 \%$ of cases.

Key words: Pancreas transplant-Surgical complications

\section{Introduction}

Over the past few years there have been at least two major factors which have improved the technical results of pancreas transplantation. First of all the ability to harvest simultaneously liver and whole pancreas with a common in situ perfusion with University of Wisconsin solution according to Starzl's procedure (Starzl 1987). Second, the reduction of technical failures and the ability to monitor rejection with the duodenopancreatic bladder drainage technique which has been popularized by Madison's group (Sollinger 1988).

In the light of these latest improvements we reviewed the pancreas and kidney transplantation programme at San Raffaele Hospital-Milan by considering the results in terms of survival and post-operative complications.

\section{Subjects and Methods}

At the University of Milan, San Raffaele Hospital, from July 1985 to January 1990, 33 simultaneous pancreas and kidney transplants were performed in 32 patients with Type 1 (insulin-dependent) diabetes mellitus and ureamia (one patient was pancreas and kidney retransplanted). Three dissynchronous transplants at the very beginning of the transplantation programme were not considered in the present analysis. In 26 cases a segmental neoprene duct-injected transplantations was performed. From October 1989, seven duodenopancreatic transplantation. with bladder drainage were performed.

Mean age of the patients was $38.7 \pm 1.3$ years, the mean duration of Type 1 diabetes $24.6 \pm 1.1$ years and the mean duration of dialysis $17.4 \pm 2.1$ months. All patients had peripheral vasculopathy, autonomic and peripheral neuropathy and retinopathy (one patient was blind).

Access was through a median laparotomy in the first 18 cases and through a bilateral paramedian $J$ incision in the latest cases.

The segmental pancreas was duct injected with neoprene $(2.5-6 \mathrm{ml})$ according to Du Bemard's technique (1978) and erafted to the iliac vessels (in 11 cases to the internal iliac artery, in 11 to the external, in three to the common and in one case to the inferior mesenteric artery).

Duodencpancreatic transplants (Sollinger 1988) were grafted in all cases to the external iliac artery. In six cases an extended iliac artery graft was used and in one case an aortic patch was available.

The mean warm ischaemia time was $42.4 \pm 13 \mathrm{~min}$ and the mean cold ischaemia time 5 hours $\pm 82 \mathrm{~min}$.

Table 1. Anticoagulative protocol

Induction Maintenance

July 85-March 88

Heparin Acenocoumarole

(PTT ratio $>2.0$ ) (PT ratio $>1.3<1.5$ )

April 88-Sept 89 (randomized study)

Dextran Dipiridamole (75 mg tid*)

(250ml/day) ASA (250 mg uid $f$ )

vs

Antithrombin III Acenocoumarole

(100 UT/h) (PT ratio $>1.3<1.5$ )

followed by Calcium Heparin

$(0.2 \mathrm{ml} \mathrm{tid*)}$

from October 89

Calcium Heparin Dipiridamole (75 mg tid*)

$(0.2 \mathrm{ml} \mathrm{tid*)}$ ASA (100 mg uid $f)$

*tid = three times daily

fuid = once daily 
To prevent graft thrombosis patients underwent an anticoagulative protocol which changed with time (Table 1)

The immunosuppressive protocol included azathioprine $\left(2.5 \mathrm{mg} \cdot \mathrm{kg}^{-1}\right.$.day1 , maintenance $1 \mathrm{mg} \cdot \mathrm{kg}^{-1} \cdot \mathrm{day}^{-1}$ ) Prednisone (10 mg.kg-1.day ${ }^{-1}$, progressively reduced to $10 \mathrm{mg}$.day), Antilymphocyte globulins (ALG) ( $8 \mathrm{mg} . \mathrm{kg}^{-1} \cdot \mathrm{day}^{-1}$ for 10 days). ALG was then withdrawn and $6-8 \mathrm{mg} \cdot \mathrm{kg}^{-}$ 1 .day ${ }^{-1}$ of cyclosporin was administered. Acute rejection episodes were treated by methylprednisolone flushes $(500 \mathrm{mg})$.

From October 1989 the immunosuppressive protocol was modified in the following way: Prednisone ( $1 \mathrm{mg}^{\mathrm{kg}} \mathrm{k}^{-1} \cdot \mathrm{day}^{-1}$, maintenance $\left.10 \mathrm{mg} \cdot \mathrm{day}\right)$. Unresponsive acute rejection episodes were treated with OKT3 monocolonal antibodies.

All patients underwent peri-operative antibiotic prophylaxis with a $3^{\text {rd }}$ generation cephalosporin and long-term prophylaxis with cotrimoxazole. Antifungal and antiviral prophylaxis were performed with ketoconazole and acyclovir.

\section{Results}

Patient, kidney and pancreas actuarial survival are shown in Figure 1. Patient 1-year and 2-year survival were respectively, 93 and $89 \%$, which remained unchanged for 5 years.

Kidney 1-year survival was still $93 \%$ while 5-year was $72 \%$ mainly due to chronic rejections.

Pancreas 1-year survival was $68 \%$, the 5-year rate was $58 \%$. Most of the failures were observed during the first 3 month period. Late failures were caused by chronic rejection. One-year and 5-year pancreas graft survival in technical successful cases were $88 \%$ and $73 \%$.

The overall mortality was $6 \%$ : one patient died of myocardial infarction with both organs functioning and one patient died of septic bleeding after the removal of both the organs.

A quarter of the transplantations had an uneventful postoperative course $(24.4 \%)$. Surgical complications were present in $51 \%$ of patients while $71 \%$ of patientsshowed rejection or complications related to immunosuppressive therapy and $43 \%$ of the cases had nonsurgical infections.

Causes of graft failure are reported in Table 2. Twenty pancreas grafts have been functioning from 5 to 66 months and in 26 cases the grafted kidney is still functioning after the same follow-up period. Pancreas function failure was due to thrombosis in seven cases $(20 \%)$ and rejection in four cases (12\%). Kidney failure was mainly secondary to chronic rejection (after a mean of 30 months). In only one case a surgical complication was the reason for loss of kidney graft function due to pancreas graft abscess.

Acute kidney rejection was observed in 19 cases and in four cases a chronic rejection ensued ( after a mean of 30 months) which led to the loss of function.

Severe acute pancreas rejection was observed in two cases. In one of them pancreas function recovered completely after treatment

Fourteen patients had one or more non-surgical infection (13 cytomegalovirus, 8 herpes simplex virus, 2 herpes zoster virus infections). In one case a Staphilococcus Aureus Endocarditis and in another a Guillain-Barre Syndrome was observed.

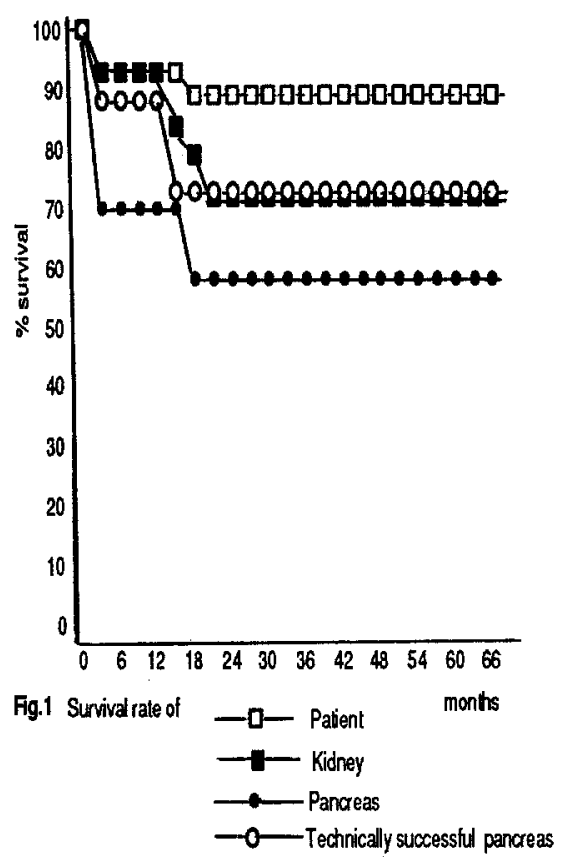

Seventeen patients $(51 \%)$ had a post-operative surgical complication. In nine cases (27\%) the surgical complication led to graft failure, in one case $(3.6 \%)$ to death and in seven cases a relaparotomy was needed (21\%).

Arterial or venous thrombosis were the most common complications (21\%)

Table 2. Causes of graft failure

Pancreas [ $n=13$ cases (39\%)]

*thrombosis $7(20 \%)$

$*_{\text {rejection } \quad 4(12 \%)}$

*primary non-function 1 (3\%)

*death with functioning graft 1 (3\%)

Kidney [ $\mathrm{n}=6$ cases $(18 \%)]$

* rejection $\quad 4(12 \%)$

*thrombosis 1 (3\%)

*death with functioning graft $1 \quad(3 \%)$

In five patients (15\%) a pancreatic fistula developed. In all cases the fistula healed in less than 2 weeks and in only one case was complicated by an infection which required a relaparotomy for peritonitis. The heavy anticoagulative protocol during the early experience lead to haemoperitoneum in two cases for which one required relaparotomy. Two patients developed a septic haemorrhagic complication which required relaparotomy.The first patient had a thrombosed 
pancreas, and a vasculitis of the anastomosis secondary to peripancreatic abscess which caused a septic bleeding. The pancreas graft was removed and the patient died on the 6th day after the relaparotomy. The second patient had a localized stump pancreatitis, complicated with infection which caused a septic bleeding.

\section{Discussion}

Pancreas transplantation together with kidney transplantation as treatment of end-stage renal failure in diabetic patients remains somewhat controversial despite reports showing an improved success rate for both organs. Overall results of pancreas graft survival are still hindered by technical failures. In North American centres technical failures were recently reduced to $5-10 \%$ due to the use of the whole pancreas-bladder drainage technique.

Our overall results, comparing the group of neoprene ductinjection and bladder drainage technique showed a $20 \%$ failure rate due to technical causes which requires further improvements.

Pancreas graft rejections were observed in five cases (in two cases early and three delayed). In only one case of the latest bladder drainage series, an early acute rejection could be clearly documented, healed and treated. The bladder drainage technique allowed monitoring of amylasuria and the performance of diagnostic fine needle aspiration biopsy. Such diagnostic techniques were essential in treating and reversing rejection.

The overall morbidity rate could be considered acceptable (51\%) but the quality of major complications which required relaparotomy is still a heavy burden for patients who are already at high risk for surgical procedures. The mortality rate is comparable to that observed in major elective surgical procedures. In our study post-operative deaths were accounted for by the critical cardiac conditions, which pointed out the need of a more severe cardiologic work-up for selection, and by surgical complications such as septic bleeding which was potentially lethal in these patients. Thus, a more aggressive post-operative surveillance and treatment for surgical complications is warranted.

Acknowledgements This study was supported by Consiglio Nazionale delle Ricerche (CNR) grant no.90.00034.PF70

\section{References}

Du Bernard JM, Traeger J, Neyra P, Touraine JL, Tranchant D, Blanc-Brunat N (1978) New method of preparation of a segmental pancreatic graft for transplantation. Trials in dogs and in man. Surgery 84:634-639

Sollinger HW, Stratta RJ, D'Alessandro AM. Kalayoglu M. Pirsch JD, Belzer FO (1988) Experience with simultaneous pancreas-kidney transplantation. Ann Surg 208:475-482
Starzl TE, Miller G, Bronik B, Makowka L (1987) An improved technique of multiple organ harvesting . Surg Gynecol Obst 165:343-348

\author{
Dr. R. Castoldi \\ Divisione Chirurgia II \\ San Raffaele Hospital \\ via Olgettina 60 \\ I-20132 Milan \\ Italy
}

